Pacific Journal of Mathematic 


\title{
ON EPIMORPHISMS TO FINITELY GENERATED MODULES
}

\author{
E. Graham Evans, Jr.
}

\begin{abstract}
Serre's theorem on projective modules says roughly that if a projective $R$ module is big enough it can map onto $R$. Forster and Swan discuss how big a free module is needed to map onto a given finitely generated module. This note examines a common generalization of these results and extends Swan's technique.
\end{abstract}

This paper follows Swan [5]. The reader is urged to refer to Swan for a more complete exposition of some of the ideas. The author is also in debt to Professor Kaplansky whose unpublished exposition of Swan's result [2] isolated one of the ideas for this paper.

Throughout the paper $R$ will be a commutative ring with identity whose maximal ideal spectrum is a noetherian space and $\Lambda$ is an $R$ algebra which is a finitely generated $R$ module. Following Swan we define $J$-Spec $(R)$ to be the set of all prime ideals of $R$ which are intersection of maximal ideas with topology the subspace topology inherited from $\operatorname{Spec}(R)$. If $M$ is a finitely generated $R$ module, then for each $p \in J$-Spec $(R)$ we define $b(p, M)=0$ if $M_{p}=0$ and

$$
b(p, M)=r+d
$$

where $r=\operatorname{dim}_{(R / p)_{p}}(M / p M)_{p}$ and $d=\operatorname{dim} J$-Spec $(R / p)$ otherwise. We also call an element $x \in M_{p}$ basic if it will serve as part of a set of generators with the minimal number of elements, i.e., if $M_{p} / R_{p} x$ requires fewer generators than $M_{p}$.

THEOREM 1. $\mathrm{R}$ a commutative ring with $J$-Spec $(R)$ a noetherian space $M$ a finitely generated $R$ module and $P$ a finitely generated projective $R$ module with rank $(P) \geqq \max b(p, M)$. Then there exists an epimorphism from $P$ to $M$.

Proof. We might as well assume that $M$ is faithful. For if $\mathfrak{a}=\operatorname{ann}(M)$, then we pass to $P / \mathfrak{a} P$ which is projective over $R / \mathfrak{a}$ with rank at least as large. Then if $p$ is a minimal prime in $J$-Spec $(R)$ such that a chain of maximal length of primes in $J$-Spec $(R)$ passes through $p, M_{p} \neq 0$ since otherwise there would exist an $s \in R-p$ with $s M=0$ contrary to $M$ being faithful. Hence $\operatorname{dim}_{(R / p)} \geqq 1$. Thus $\operatorname{rank}(P) \geqq d+1$ where $d=\operatorname{dim} J$-Spec $(R)=\operatorname{dim} M$-spec $(R)$. Hence by Serre's theorem $P=R \oplus P^{\prime}$. We define an epimorphism $f$ from $P$ to $M$ by $f((1,0))=m$ where $m$ is an element of $M$ which is basic 
at all $p^{\prime}$ such that $b\left(p^{\prime}, M\right)$ is maximal. (See Swan [5, p 320] or below for details.) Then $\operatorname{Max}(b(p, M /(m)))$ is one less. Hence $P^{\prime}$ maps onto $M /(m)$ by induction. $P^{\prime}$ is projective. Hence that map lifts to $g: P^{\prime} \rightarrow M$. Let $f((0, x))=g(x)$. Then $f$ is clearly an epimorphism as desired.

REMARKs. This, of course, extends to the case of $P$ and $M$ being finitely generated $\Lambda$ modules since both Serre's and Swan's theorems are true in that case also. See [4, Theorem 11. 2 p. 171] and [5, Theorem 2, p. 320].

CoRollary 2. $R$ as above $P$ a projective $R$ module of rank $\geqq r+d$ where $d=\operatorname{dim} J-\operatorname{Spec}(R)$. $Q$ any projective of rank $r$. Then $P$ is isomorphic to $P^{\prime} \oplus Q$.

\section{Proof. Clear.}

TheOREM 3. $R$ commutative with $J$-Spec $(R)$ a noetherian space. $M$ a finitely generated $R$ module. $N$ any $R$ module such that a direct sum of some number of copies (finite will of course suffice) of $N$ maps onto $M$. Then if $n=\max b(p, M)$ a direct sum of $n$ copies of $N$ will map onto $M$.

Proof. The key result needed from Swan is [5, p. 320 remark after Proposition 3] which states that the number of primes where $b(p, M)$ is maximum is finite.

We proceed to construct $f: \sum_{i=1}^{n} N \rightarrow M$ on each component in such a way that $\operatorname{Max} b\left(p, M /\right.$ image $\left.\left(\sum_{i=1}^{j} N \rightarrow M\right)\right) \leqq \max b(p, M)-j$ until $\max b\left(p, M\right.$ /image $\left.\left(\sum_{i=1}^{j} N \rightarrow M\right)\right)=0$ in which case image $\left(\sum_{i=i}^{j} N \rightarrow M\right)=M$. Then we finish the epimorphism by sending the remaining components anywhere.

Suppose $f$ has been contructed on the first $j$ components $(j=0$ is allowed). Let the image of $\sum_{i=1}^{j} N=I_{j}$. Then if $\max b\left(p, M / I_{j}\right)=0$ we are done. Otherwise $\max b\left(p, M / I_{j}\right)>0$. Let $p_{1}, \cdots, p_{m}$ be all the primes where $b\left(p, M / I_{j}\right)$ is maximal. Consider the submodules $p_{i} * I_{j}=\left\{m \mid \exists s \in R-p_{i} \quad\right.$ with $\left.\quad s m \in p_{i} M+I_{j}\right\} . \quad p_{i} * I_{j} \neq M \quad$ since $\left(M / I_{j}\right)_{p_{i}} \neq 0$. Furthermore an element $m \in M$ is part of a minimal generating set for $\left(M / I_{j}\right)_{p_{i}}$ if and only if $m \notin p_{i} * I_{j}$. (This is an easy consequence of Nakayama's lemma.) Since a direct sum of copies of $N$ maps onto $M$ there is some map $f_{i, j} ; N \rightarrow M$ such that image $f_{i, j} \not \subset p_{i} * I_{j}$. We will achieve our objective if we can find an $f_{j}: N \rightarrow M$ with image $f_{j} \not \subset p_{i} * I_{j} \cup \cdots \cup p_{m} * I_{j}$. We prove this by induction on $m$. The case $m=1$ is already done. We arrange the primes $p_{1}, \cdots, p_{m}$ so that $p_{i}$ is minimal among $p_{1}, \cdots, p_{i}$. We assume we have an $f_{j}$ 
that works for $p_{1}, \cdots, p_{s}$. Then we want one working for $p_{1}, \cdots, p_{s+1}$. If $f_{j}$ does, fine. Otherwise image $f_{j} \subset p_{s+1} * I_{j}$. Pick

$$
r \in p_{1} \cap \cdots \cap p_{s}-p_{s+1}
$$

which exists since $p_{s+1}$ cannot contain $p_{i}$ if $i<s+1$. Then I claim $f_{j}+r f_{s+1, j}$ works. It works at $p_{s+1}$ since image $f_{j} \subset p_{s+1} * I_{j}$ while $r f_{s+1} \not \supset p_{s+1} * I_{j}$. Hence image $f_{j}+r f_{s+1, m} \not \supset p_{s+1} * I_{j}$. On the other hand at $p_{i}$ for $i<s+1$ we have image $r f_{s+1, j} \subset p_{i} * I_{j}$ while image $f_{j} \not \subset p_{i} * I_{j}$. Hence image $f_{j}+r f_{s+1, m} \not \subset p_{i} * I_{j}$. This completes the proof.

REMARKs. The theorem as it stands is false for general $\Lambda$. For if $\Lambda=n$ by $n$ matrices over a field, $N=a$ column, $M=\Lambda$. Then at least $n$ copies of $N$ are needed to map onto $M$ but $\max b(p, M)=1$. The difficulty in the proof is that in the non-commutative local case the set of not basic elements are not a submodule. The proof above uses heavily that the not basic elements are a submodule locally. In fact the $p_{j} * I_{j}$ are exactly the elements of $M$ which are not basic in $\left(M / I_{j}\right)_{p_{i}}$. I conjecture that if $M$ is generated by $n$ elements over $R$ and $q$ is the biggest integer $\leqq$ the square root of $n$ that $q \operatorname{maxb}(p, M)$ would work.

Another difficulty with this result is if $N$ were free on a large number of generators then certainly we should be able to notice this and get a much better bound which this theorem cannot detect. Perhaps one could define a function $b(p, N, M)$ which would use the number of copies of $N_{p}$ needed to map onto $M_{p}$. A theorem of this type might give back Serre's theorem except, for general $N$, one certainly needs the hypothesis that a sum of $N$ 's maps onto $M$.

We recall that in the category of $R$ modules a generator is any module such that a sum of copies of it maps onto $R$. Or equivalently if for every module $M$ and submodule $N$ with $N \neq M$. There is a map $f: G \rightarrow M$ with image $f \subset N$. Theorem 3 shows for $R$ a module $N$ is a generator if and only if a sum of $d$ copies of $N$ maps onto $R$ where $d=\operatorname{dim} m$-Spec $(R)$.

\section{REFERENCES}

1. O. Forster, Uber die anzahl der Erzeugenden einen Ideals in einem Noetherschen Ring, Math. Zeit., 84 (1964), 80-87.

2. I. Kaplansky, Topics in Commutative Ring Theory, mimeographed notes University of Chicago 1969.

3. J. P. Serre, Modules Projective et Espaces Fibres a Fibre Vectorielle, Seminaire P. Dubreil, Paris (1958), 23-01-23-18. 
4. R. G. Swan, Algebraic K-theory, Springer-Verlag, Berlin 1968.

5. - The Number of Generators of a Module, Math. Zeit., 102 (1967), $318-322$.

Received August 5, 1970. This work was supported under grant No. GP-9661.

UNIVERSITY OF CALIFORNIA AND

MassaChusetTs Institute of TeChNOLOGY 


\section{PACIFIC JOURNAL OF MATHEMATICS}

\section{EDITORS}

H. SAMELSON

Stanford University

Stanford, California 94305

C. R. Новву

University of Washington

Seattle, Washington 98105
J. DUGundJI

Department of Mathematics

University of Southern California

Los Angeles, California 90007

RICHARD ARENS

University of California

Los Angeles, California 90024

\section{ASSOCIATE EDITORS}
E. F. BECKENBACH
B. H. NeumanN
F. WOLF
K. YOSHIDA

\section{SUPPORTING INSTITUTIONS}
UNIVERSITY OF BRITISH COLUMBIA
CALIFORNIA INSTITUTE OF TECHNOLOGY
UNIVERSITY OF CALIFORNIA
MONTANA STATE UNIVERSITY
UNIVERSITY OF NEVADA
NEW MEXICO STATE UNIVERSITY
OREGON STATE UNIVERSITY
UNIVERSITY OF OREGON
OSAKA UNIVERSITY
UNIVERSITY OF SOUTHERN CALIFORNIA
STANFORD UNIVERSITY
UNIVERSITY OF TOKYO
UNIVERSITY OF UTAH
WASHINGTON STATE UNIVERSITY
UNIVERSITY OF WASHINGTON
AMERICAN MATHEMATICAL SOCIETY CHEVRON RESEARCH CORPORATION TRW SYSTEMS
NAVAL WEAPONS CENTER 


\section{Pacific Journal of Mathematics}

\section{Vol. 37, No. $1 \quad$ January, 1971}

Gregory Frank Bachelis and Haskell Paul Rosenthal, On unconditionally

converging series and biorthogonal systems in a Banach space .........

Richard William Beals, On spectral theory and scattering for elliptic

operators with singular potentials .........................

J. Lennart (John) Berggren, Solvable and supersolvable groups in which every element is conjugate to its inverse ........................ 21

Lindsay Nathan Childs, On covering spaces and Galois extensions ..........

William Jay Davis, David William Dean and Ivan Singer, Multipliers and

unconditional convergence of biorthogonal expansions..............

Leroy John Derr, Triangular matrices with the isoclinal property ............

Paul Erdős, Robert James McEliece and Herbert Taylor, Ramsey bounds for

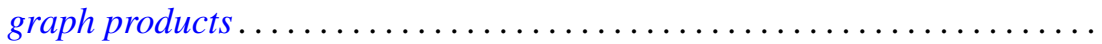

Edward Graham Evans, Jr., On epimorphisms to finitely generated

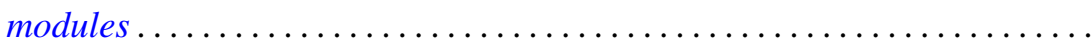

Hector O. Fattorini, The abstract Goursat problem ................. 51

Robert Dutton Fray and David Paul Roselle, Weighted lattice paths .........

Thomas L. Goulding and Augusto H. Ortiz, Structure of semiprime $(p, q)$

radicals ...........................................

E. W. Johnson and J. P. Lediaev, Structure of Noether lattices with join-principal maximal elements ....

David Samuel Kinderlehrer, The regularity of minimal surfaces defined over

slit domains

Alistair H. Lachlan, The transcendental rank of a theory. .

Frank David Lesley, Differentiability of minimal surfaces at the boundary ...

Wolfgang Liebert, Characterization of the endomorphism rings of divisible torsion modules and reduced complete torsion-free modules over complete discrete valuation rings....

Lawrence Carlton Moore, Strictly increasing Riesz norms.

Raymond Moos Redheffer, An inequality for the Hilbert transform ...

James Ted Rogers Jr., Mapping solenoids onto strongly self-entwined,

circle-like continua..........................

Sherman K. Stein, B-sets and planar maps ................... 217

Darrell R. Turnidge, Torsion theories and rings of quotients of Morita

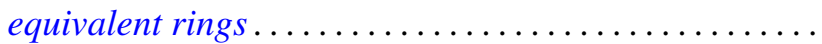

Fred Ustina, The Hausdorff means of double Fourier series and the principle of localization ................................

Stanley Joseph Wertheimer, Quasi-compactness and decompositions for arbitrary relations.

Howard Henry Wicke and John Mays Worrell Jr., On the open continuous images of paracompact $\check{C}$ ech complete spaces... 